\title{
OS ESTUDOS DE LINGUAGEM DO IDOSO NESTE ÚLTIMO SÉCULO
}

Lenisa Brandão ${ }^{1}$

Maria Alice de Mattos Pimenta Parente ${ }^{2}$

\section{Resumo}

A linguagem do idoso, cada vez mais, tem sido o foco de pesquisas da psicologia e da lingüística que buscam identificar transformações e detectar as causas de possíveis mudanças que ocorrem no processo de envelhecimento. Este artigo revisa importantes estudos sobre a linguagem do idoso, enfocando as descobertas, as controvérsias e as hipóteses da manutenção ou do declínio de funções lingüísticas na velhice. A pesquisa neste campo ainda está em período inicial, porém a interação entre disciplinas é crescente. Além disso, os estudos começam a enfocar as funções que são preservadas ou que evoluem com o avanço da idade e surgem tendências para o desenvolvimento de perspectivas futuras de intervenção durante o processo de envelhecimento. As pesquisas vem demonstrando que independente dos resultados demonstrarem declínios, habilidades preservadas ou superiores, a investigação deve retornar com frutos positivos para a vida dos idosos.

Palavras-chave: Linguagem; Cognição; Envelhecimento.

\section{Abstract}

Cognitive psychology is increasingly expanding its studies about language and the processes of aging. The studies investigating the cognitive changes that accompany aging rise questions about the stability of language or the apearence of deficits of lingüistic functions in the aged. A great number of findings, controversials and hipothesis in this field are presented in the studies gathered in the present paper. Although research about language and aging is in its begining, there is a growing interest in the interaction of multiple perspectives, the preservation and evolution of some cognitive functions, and trends for cognitive intervention for older adults, with a greater emphasis on producing productive results in older adults lives.

Key words: language; cognition; aging.

${ }^{1}$ Fonoaudióloga, Mestranda em Psicologia do Desenvolvimento, UFRGS. email- lenisa@lycos.com ${ }_{2}^{2}$ Professora Doutora Departamento de Psicologia do Desenvolvimento, Instituto de Psicologia, UFRGS.

Estud. interdiscip. envelhec., Porto Alegre, v.3, p.37-53, 2001. 


\section{Introdução}

O estudo da linguagem humana não é exclusivo de uma área científica. Ao contrário, muitas disciplinas, com suas diferentes perspectivas têm procurado entender e explicar essa importante atividade para o desenvolvimento do homem. O mesmo ocorre com os estudos da linguagem do idoso, que buscaram, inicialmente, identificar transformações e detectar as causas de possíveis mudanças que ocorrem no processo de envelhecimento. Entretanto, recentemente, em vista do aumento crescente de expectativa de vida, os trabalhos sobre cognição do idoso, incluindo aqueles sobre linguagem, dirigem-se, também, à promoção de uma melhor qualidade de vida nessa etapa significativa.

Dentro de uma perspectiva histórica, inicialmente a Lingüística teve uma maior influência nos estudos sobre a linguagem dos idosos, que seguiram a evolução dos enfoques da própria Lingüística, ou seja, do interesse pela sílaba ao discurso.

A fundação da lingüística moderna, no início do século, inseriu-se dentro da corrente científica do estruturalismo, que caracterizou-se pela descrição sistemática, parcial ou total das estruturas da linguagem (fonologia, lexicologia e semântica), focalizando a palavra isolada, ou seja a formação sonora da palavra e a denominação (Beneviste, 1995). Embora o estruturalismo não represente hoje a corrente mais utilizada nas pesquisas em Línguiística, vários estudos verificam os aspectos formais da linguagem do idoso, avaliando fatores relacionados à sintaxe, semântica e fonologia (Orlandi, 1990).

Estudos que analisam os aspectos fonológicos da linguagem na velhice demonstram um déficit na compreensão de fonemas distorcidos ou apresentados com ruído de fundo. Este déficit muitas vezes está ligado a uma diminuição da audição. Além disso, outros trabalhos observaram que a produção de fonemas no idoso é distinta, sendo identificada por ouvintes como menos clara, apesar de não dificultar demasiadamente sua compreensão (Ryan; Laurie, 1990). No entanto, também é observado que, freqüentemente, os idosos utilizam estratégias mais ou menos eficientes para manejar com estas dificuldades, como a utilização de habilidades lingüísticas do tipo top-down, em que o discurso como um todo e o contexto em que ele ocorre é o principal fator considerado na compreensão (Stine; Wingfield; Poon, 1989). 
Entretanto, a análise dos aspectos semânticos e lexicais da linguagem do idoso mostra que nem sempre são encontradas perdas ou prejuízos em relação a faixas etárias mais jovens. Apesar de um provável declínio na produção de palavras, caracterizada pelo aumento de comportamentos de tipo “. . . palavra na ponta da língua.” (Burke; Harrold, 1988), o vocabulário permanece estável ou mesmo evolui, especialmente nos idosos que apreciam a leitura (Obler, 1989; Salthhouse, 1987). Desta forma, podemos observar uma evolução dissociada, na qual perdas de rapidez e de articulação, que podem ocasionar perdas na fluência e na inteligibilidade das mensagens de idosos, não refletem a evolução semântica, provavelmente ocasionada pela maior experiência lingüística com o decorrer da vida.

Outros estudos não se preocuparam com o envelhecimento, mas sim em caracterizar a forma dos idosos em utilizar a linguagem, enquanto um grupo social distinto. Pretti (1991) abordou as características do léxico no discurso do idoso, mais especificamente, aquelas que evidenciavam marcas lexicais de espaço e tempo. Este trabalho demonstrou o aparecimento constante de informações sobre o passado, através do surgimento de determinadas expressões, vocábulos, formas de tratamento e estruturas formulaicas antigas. Arcaísmos gírios, ou seja, gírias antigas, apareceram nos discursos, tais como coió (rapaz conquistador) e vassourinha (moça namoradeira), que surgiram com freqüência. Também foi observado o uso de expressões formulaicas, que são frases-feitas, provérbios e refrões, como "de grão em grão a galinha enche o papo", para as quais os idosos pareceram ser bastante afeitos, sendo que muitas dessas expressões remontavam à sua infância e juventude. Algumas formas de tratamento próprias do idoso também puderam ser observadas em idosos mais velhos, verificando-se maior formalidade na linguagem. Pretti (1991) concluiu que, com exceção das formas de tratamento, considerável parte do vocabulário do idoso é atualmente desconhecida e provoca um constante processo de explicação na sua fala, quando se dirige a interlocutores jovens. Esse constante processo de explicação pode prejudicar a fluência do discurso, que se caracteriza por saídas e voltas ao tópico, podendo ocorrer a saída sem retorno ao assunto original, que pode estar associada às dificuldades de memória do idoso. O autor afirma que estas explicações fornecidas pelo 
idoso, relacionadas à linguagem, parecem não configurar um problema para ele, que expressa prazer em transmitir conhecimentos aos jovens.

Nos anos 70, a lingüística sai da descrição exclusiva das estruturas da linguagem para observar o seu uso. Surge a Pragmática, uma corrente preocupada com a significação estabelecida no uso concreto da linguagem por falantes reais. Uma das principais vertentes da Pragmática considera o usuário em uma relação de interlocução com o outro, desenvolvendo estudos que abordam o contexto de comunicação. Desses estudos, surgiram a Análise Conversacional, os Atos de Fala e a Teoria da Enunciação. Na pragmática conversacional, o significado é considerado em função da intenção do locutor e do reconhecimento dessa intenção pelo ouvinte. Já a teoria dos atos de fala considera que a linguagem é utilizada para realizar vários tipos de ações, valorizando a ação que está implícita na fala do locutor (Searle, 1969). A teoria da enunciação salienta o sujeito locutor buscando compreender o processo subjetivo de enunciação, de acordo com as formas que o sujeito usa para registrar sua própria marca naquilo que diz.

Estudos abordando a linguagem do idoso sob estes prismas atuais vem sendo apresentados de forma crescente na literatura. Boden; Bielby (1983) realizaram um estudo exploratório que utilizou a análise de conversação como método para verificar a significância da estória de vida ou narrativa pessoal na vida diária do idoso. Conversas entre díadas de idosos e díadas de jovens foram comparadas, demonstrando que a lembrança do passado é freqüente nas conversas entre idosos, aparecendo bem menos na interação de jovens. De acordo com o estudo de Pretti (1991), que abordou o tópico discursivo e a fluência entre outros fatores da linguagem no que se refere às narrativas conversacionais, o discurso de idosos não difere fundamentalmente do discurso de indivíduos de outras faixas de idade. O autor afirma, no entanto, que as narrativas ocorrem com grande frequiência na fala de indivíduos mais velhos, dada a tendência natural dos idosos em tornarem-se "contadores de estórias". No estudo de Pretti, o discurso dos idosos apresentou, com frequiência, a narrativa de casos pessoais como tópico, despertando o interesse do ouvinte. A categoria tempo percorreu a construção dos tópicos, que se bipartiram entre o passado e o presente. Segundo o autor, o passado é utilizado para a análise 
do presente, sendo mais importante para o falante idoso pois ele crê e pretende demonstrar que os valores de antigamente são superiores aos de hoje. Assim, ele apresenta o objetivo de preservar sua imagem social através da linguagem. De acordo com Pretti (1991), os idosos não demonstraram um déficit em seus discursos, mas revelaram habilidade em conduzir, quebrar e mudar de tópicos, visando a oposição desejada entre o passado e o presente.

Recentemente, observa-se que os estudos sobre linguagem, não recebem a influência teórica e metodológica de apenas uma área de conhecimento. Neste sentido, a Psicolingüística, coordena conhecimentos da Lingüística e da Psicologia, focalizando a atividade da linguagem, seus processos e formas de utilizá-la.

\section{A Psicologia e os Estudos sobre a Linguagem dos Idosos}

A influência da Psicologia nos estudos da linguagem do idosos acompanha quatro perspectivas teóricas da própria disciplina: a perspectiva do desenvolvimento, a perspectiva psicométrica, a perspectiva do processamento das informações e a perspectiva psico-social (Pratt; Norris, 1994).

Estudos dentro da perspectiva do desenvolvimento, utilizando-se do modelo de estágios de Piaget, sugeriram que adultos mais velhos apresentavam desempenho menos adequado do que adultos jovens, considerando as operações formais descritas por Piaget (Bielby; Papalia, 1975; Looft, 1972). Estes achados foram interpretados como indícios de uma regressão cognitiva. No entanto, os resultados foram questionados, devido à falta de um delineamento longitudinal que fundamentasse de forma consistente a hipótese de um declínio cognitivo do idoso. Os estágios de desenvolvimento cognitivo propostos por Piaget se estancam no período das operações formais, encerrando o desenvolvimento da inteligência na adolescência. No entanto, desde 1980, a abordagem de Piaget vem sendo utilizada para propor a etapa pós-formal (Commons; Richards; Armon, 1984). Segundo os autores que seguem esta linha, ocorrem estágios posteriores de desenvolvimento cognitivo após o período das operações formais. Os estudos sobre a linguagem do idoso, nesta perspectiva, atribuem ao idoso a característica do egocentrismo, descrito por Piaget (Rubin, 1974); outros 
sugerem uma regressão caracterizada por um padrão inverso ao da aquisição da linguagem por crianças, isto é, as habilidades lingüísticas mais complexas, adquiridas mais tarde pela criança, são as primeiras a desaparecer no idoso (Kemper, 1988).

Uma abordagem de etapas rígidas não permite a noção de que possam haver evoluções diferenciadas em determinados aspectos cognitivos, causando as dissociações já mencionadas anteriormente. Mesmo que alguns aspectos do comportamento lingüístico, principalmente o articulatório e a fluência expressiva, mostrem um declínio, a utilização freqüente de diferentes formas lingüísticas pode promover o enriquecimento semântico e lexical durante o envelhecimento. Mac Kay; Abrams; Pedroza (1999), num estudo sobre a detecção e a reprodução de erros ou acertos ortográficos, sustentaram a hipótese de uma assimetria no desempenho de idosos quanto à compreensão e à produção, sugerindo que os processos de compreensão permanecem estáveis, enquanto os processos de produção apresentam declínios. Desta forma, parece evidente que há processos cognitivos relacionados à linguagem mais vulneráveis ao envelhecimento do que outros.

A perspectiva psicométrica está centrada no uso de testes padronizados que são elaborados através de procedimentos controlados e delineados para investigar diferenças individuais de desempenho cognitivo nos diferentes estágios da vida. Analisando-se historicamente os estudos desta corrente, verifica-se que grande parte das pesquisas nesta linha defenderam hipóteses de declínio na inteligência do idoso. A sugestão da existência de uma inteligência unitária que, em bebês, seria indiferenciada, tornando-se diferenciada na vida adulta e sofrendo um retrocesso caracterizado pela indiferenciação na velhice, foi uma das hipóteses testadas na perspectiva psicométrica (Balinsky, 1941). Estes achados foram veementemente criticados, encontrando apoio insuficiente para sua fundamentação. Outra proposição da psicometria é a existência de uma inteligência fluida, que aporta habilidades em tarefas novas, e uma inteligência cristalizada, que é responsável por tarefas que envolvem informação cultural já aprendida. Pesquisas que recorrem a esta proposição consideram que a inteligência fluida sofre declínio na velhice, enquanto a inteligência cristalizada se mantém constante, podendo até mesmo desenvolver acréscimos 
nesta fase da vida (Horn, 1982). Evidências de estudos mais recentes (Cuningham; Tomer, 1990) demonstraram a existência de um declínio menor da inteligência fluida, ocorrendo em idades mais avançadas do que o previsto pelo estudo de Horn (1982). A idéia das habilidades fluidas e cristalizadas apoia estudos com a hipótese de que a linguagem permanece intacta na velhice, pois a linguagem é considerada uma habilidade cristalizada.

A perspectiva psico-social contribui com a ênfase na diversidade individual e no contexto para explicar o modo como as habilidades manifestamse ao longo da vida e se propõe a descrever o ambiente social no qual o desempenho cognitivo do sujeito está inserido. Nesta perspectiva, as habilidades cognitivas são modeladas pelo contexto histórico e cultural. Assim, esta corrente direciona grande parte dos estudos sobre a linguagem do idoso para a relação deste com a sociedade, verificando a maneira como esta se comunica com o idoso e argumentando que esta maneira de se comunicar produz um impacto negativo na percepção do idoso sobre suas próprias habilidades lingüísticas. Levando em conta aspectos sociais e culturais, esta perspectiva contou com a contribuição da sociologia e da antropologia, bem como - mesmo que pareça paradoxal - com a contribuição da inteligência artificial e da neuropsicologia. Este intercâmbio multidisciplinar propicia o nascimento da psicologia do discurso, denominada também, a segunda revolução cognitiva (Harré; Gillett, 1994). A análise de discurso vem possibilitar o estudo da significação nos processos mentais, propondo a idéia de que o significado não tem origem apenas nas regras internas da mente. Este é o ponto de encontro de uma vasta gama de influências estruturais que incluem os conceitos disponíveis nos discursos e nos contextos em que estes são desenvolvidos. O uso da análise de discurso provém do pensamento de que não é possível definir a mente de modo isolado. Através deste método, os estudos da linguagem pretendem contribuir com a investigação da relação da linguagem e das condições de produção do discurso, ou seja, o falante, o ouvinte, o contexto da comunicação e o contexto histórico-social.

Por outro lado, a linguagem - especialmente a linguagem discursiva não é mais considerada uma atividade mental isolada, mas nela confluem diferentes processos mentais, tais como, tipos de raciocínio, formação de 
estratégias, arquivo e produção de informações. Assim, a compreensão funcional da linguagem gerou uma diversidade de estudos em Psicolingüística, englobando três principais áreas: a) o estudo da produção e compreensão da linguagem; b) o estudo das funções cognitivas da linguagem; e c) o estudo da linguagem em seus aspectos evolutivos e patológicos (Belinchón; Rivière; Igoa, 1996). Estes três ramos têm contribuído de forma crescente com estudos sobre a linguagem do idoso.

Os estudos que se interessam pela análise dos processos mentais no envelhecimento têm se caracterizado, em grande parte, pela investigação do processamento de informação do idoso. Os achados dessas pesquisas sugerem o declínio de algumas funções cognitivas, como atenção, resolução de problemas e memória. Esta última tem sido exaustivamente estudada, destacando-se pesquisas sobre a memória de trabalho dos idosos. Resultados destes estudos indicam que ocorre uma diminuição da habilidade de estocar informações a curto prazo para a resolução de problemas, fornecendo a explicação de que há uma diminuição espacial deste sistema de capacidade limitada (Light, 1991). Explicações temporais também são fornecidas com relação ao declínio de funções cognitivas do idoso. Cerella (1990) e Salthhouse (1985) referem uma diminuição na velocidade de processamento cognitivo do idoso, promovendo um desempenho menos eficiente devido ao reduzido tempo de manutenção de informações necessárias para a resolução de problemas. Críticas à perspectiva do processamento das informações têm consistido no questionamento da pesquisa dos déficits cognitivos em laboratório. Tais críticas levantam a idéia de que a sugestão de déficits contrasta com evidências de estudos que investigam o idoso em situações de vida real, encontrando desempenhos mais funcionais e em certos casos, considerados superiores. Em resposta a estas críticas, a perspectiva do processamento das informações tem incluído estudos que não envolvem somente experimentos de laboratório, mas investigam a cognição do idoso em situações de vida real. Além disso, surgem explicações que introduzem o termo "compensação", para dar conta da manutenção do nível de desempenho competente, apesar da diminuição de algumas habilidades, através da reorganização das estratégias para realizar uma tarefa (Pratt; Norris, 1994). 
A diminuição de algumas habilidades cognitivas na velhice, pode influir no declínio da linguagem no idoso. As evidências de déficits na memória de trabalho dão suporte a esta idéia, pois a memória de trabalho exerce um papel importante no processamento da linguagem (Kemper, 1988). Além dessas evidências, a proposição de que há uma diminuição da velocidade no funcionamento cognitivo prevê uma piora no desempenho lingüístico (Salthouse, 1991). De acordo com Woodruff-Pak (1997), muitas investigações têm fornecido suporte à hipótese de que déficits na linguagem do idoso resultam do declínio de processos cognitivos não lingüísticos. Dessa forma, a origem de problemas na linguagem do idoso não está ligada à alterações focais das estruturas especializadas no processamento lingüístico. De acordo com a perspectiva do processamento das informações, as causas das mudanças no desempenho lingüístico do idoso resultam de problemas na atenção, percepção, velocidade, memória e funções executivas do lóbulo frontal do cérebro.

Pesquisas sobre a produção do discurso de indivíduos idosos demonstraram que, em conversações que têm tópicos autobiográficos, há um aumento de informações pessoais irrelevantes e uma diminuição da coerência na fala, comportamento, este, que é denominado "verbosidade fora de tópico" (Arbuckle; Gold, 1993). Estes dados levantaram um debate acirrado entre as pesquisas que têm se posicionado a favor da hipótese do déficit de inibição, ou seja, da suposição de transtornos não lingüisticos, e os trabalhos que defendem a hipótese da mudança pragmática.

A hipótese do déficit de inibição postula que uma minoria de idosos saudáveis sofre um declínio específico no mecanismo de inibição, considerado uma função executiva do lobo pré-frontal do cérebro, fundamental na expressão e compreensão da linguagem, além de outras habilidades. Segundo os autores que defendem esta hipótese, a verbosidade fora de tópico parece estar associada a esta dificuldade de inibir e "clarear" a memória de trabalho de informações processadas previamente. Os achados dessa corrente demonstram que os idosos produzem fala mais prolongada e irrelevante ao assunto (Gold; Andres; Arbuckle; Schwartzman, 1988; Arbuckle; Gold, 1993; Gold; Arbuckle, 1995; Arbuckle; Nohara-Le Claire; Pushkar, 2000). 
Por outro lado, a hipótese pragmática postula que a intenção comunicativa é determinante na qualidade e no estilo do discurso. Através deste ponto de vista, a competência cognitiva não estaria por trás do estilo de discurso considerado verboso e fora de tópico pelos autores que defendem a hipótese do déficit inibitório. Segundo os defensores da hipótese da mudança pragmática, o determinante da mudança estaria no contexto social e identidade do falante. Os principais argumentos utilizados pelos defensores da hipótese pragmática para criticar a hipótese do déficit inibitório, referem-se às evidências de estabilidade de várias habilidades lingüísticas no idoso, especialmente aquelas relacionadas à tarefas de compreensão. Além destes dados, estes autores questionam o não surgimento de verbosidade fora de tópico em diferentes tarefas, como descrições de figuras. Desta forma, os resultados podem ser atribuídos à fatores psicossociais.

Burke (1997) e James (1998) argumentam que uma melhor qualidade é atribuída às estórias contadas por idosos . Ao examinar as narrativas dos idosos, Pretti (1991) afirma que os idosos tendem a falar muito, geralmente referindose ao passado. Apesar dos tópicos serem guiados pelo seu interesse pessoal, talvez mais do que pelo interesse de seu interlocutor, o discurso do idoso geralmente é considerado interessante pelo ouvinte. Segundo este autor, o sucesso nas narrativas deve-se ao grau de originalidade: quanto mais inusitados são os fatos contados, tanto maior a atenção do ouvinte ao discurso narrativo. Esta característica é, de certa forma, uma condição que favorece a ativação na memória episódica, mais preservada do que a memória de trabalho. Justamente a característica de contar fatos inusitados, mais fixados na memória, é que valoriza o discurso do idoso. Como afirma Pretti (1991), “. . . eles fazem desfilar perante o ouvinte cenas, fatos públicos, episódios familiares, tipos humanos que podem remontar a mais de meio século, devido ao próprio mecanismo da memória que possibilita aos idosos lembrar com mais facilidade cenas da infância e da juventude." (p.108).

Um dos argumentos utilizados por estudiosos que defendem a hipótese pragmática é o de que os processos de compreensão, que supostamente seriam afetados pelo déficit inibitório, parecem estar intactos na linguagem do idoso (Burke, 1997). Porém, apesar de existirem estudos que demonstram a 
preservação de habilidades relacionadas à compreensão, há trabalhos que indicam o contrário, postulando que a compreensão é afetada pelo envelhecimento. As controvérsias não param por aí, pois, como mencionado anteriormente, existem pesquisadores que defendem a hipótese de uma assimetria no declínio da linguagem do idoso, afirmando que os processos de produção são mais afetados do que os de compreensão (Mac Kay; Abrams; Pedroza, 1999).

Os achados de algumas pesquisas têm demonstrado que o desempenho de idosos não apresenta diferenças significativas do desempenho de jovens na compreensão, especificamente na tarefa de resumir um texto. Segundo estes autores, a tarefa de recontar estórias é uma atividade que envolve recursos da memória de trabalho e da memória episódica. No entanto, as pesquisas sugerem diferenças na análise de ênfases nas narrativas, demonstrando que os jovens preferem relatar a seqüência de ações e os idosos encadeiam os fatos de forma subjetiva. Devido a uma redução da memória de trabalho, os idosos utilizamse mais das informações armazenadas na memória episódica, deixando transparecer suas representações mentais (Parente; Capuano; Nespoulous, 1999; Parente; Saboskinski; Ferreira; Nespoulous, 1999). A idéia de que o idoso apresenta uma versão mais subjetiva em seu discurso do que o jovem também é sustentada pela Teoria da Seletividade Sócio-Emocional. De acordo com Cartensen,; Isaacowitz; Charles (1999), o tempo exerce um papel importante na seleção de metas sociais. O idoso apresenta uma percepção de tempo diferente daquela apresentada pelo jovem, conferindo uma certa prioridade às metas relacionadas às emoções, que se reflete no desejo de encontrar o sentido da vida e a intimidade emocional. Estas características sócio-emocionais do idoso são demonstradas no seu discurso, predominando a atribuição de um papel mais destacado à emoção em suas narrativas.

Apesar de alguns autores evidenciarem um declínio na compreensão do idoso, citando dificuldades no relato coerente de estórias (Juncos-Rabadán, 1996; Cohen,1979), muitos trabalhos sustentam a idéia de que parece não haver diferenças qualitativas significativas entre o processamento do discurso do idoso e do jovem (Stine; Wiengfield, 1990; Parente; Capuano; Nespoulous, 1999). No entanto, os autores afirmam que é provável que as diferenças de idade 
possam aparecer no processamento de discursos quando a demanda processual é alta. A capacidade reduzida de armazenamento na memória de curto prazo não explica todas as diferenças. Os autores sugerem que o idoso apresenta dificuldades em criar uma representação coerente do discurso, quando ocorre necessidade de utilização de memória de trabalho. Nesse sentido, Paul (1996) demonstrou que a detecção de falhas nos mecanismos inibitórios depende dos processos que exigem controle de atenção mais preciso, ou seja aqueles que são iniciados e gerados internamente pelo idoso mas não dos processos realizados sob o uso de dados externos, já existentes. Segundo o autor, é possível que as diferenças de idade surjam, assim que a tarefa passe a exigir que os sujeitos procurem, na memória de trabalho, as relações semânticas entre o contexto e a tarefa. Também Kemtes; Kemper (1999) observaram que os idosos podem ter dificuldades em tarefas que envolvem a coordenação do processamento simultâneo de: (1) atividades lingüísticas complexas; (2) armazenamento temporário de informações em estágios de processamento imediatos e (3) supervisão dessas atividades.

\section{Conclusões}

As controvérsias, dúvidas e mistérios que rondam os estudos a respeito da linguagem do idoso demonstram que este é um campo que ainda necessita evoluir muito, mas que está em franco crescimento e tem caracterizado-se pelo enfoque interdisciplinar. Certamente, é necessário avançar ainda mais para desvendar a cognição do idoso. Na busca por respostas às suas perguntas, o pesquisador que se ocupa deste campo deve voltar-se para a valorização do idoso. Independente dos resultados demonstrarem declínios, habilidades preservadas ou superiores, a investigação deve retornar com frutos positivos para os sujeitos.

A medida que o entendimento sobre a cognição do idoso avança, a posição dos pesquisadores começa a convergir para a idéia de que os estudos devem mover-se em direção a atitudes produtivas, abandonando a simples constatação de declínios. De acordo com Birren; Deutchman (1991), é necessário realizar uma intervenção que possibilite ao idoso desenvolver a compreensão e a valorização do self. O idoso, estando em um estágio mais 
avançado da vida, necessita encontrar o sentido de sua vida para o mundo, adaptar-se ao envelhecimento e realizar escolhas positivas para si mesmo. A proposta destes autores é o uso das narrativas auto-biográficas, que são fonte de prazer para o idoso e ao mesmo tempo possibilitam seu desenvolvimento psicossocial. Wood (2000), em um estudo que investigou a auto-eficácia cognitiva e a memória de trabalho em um programa de treinamento cognitivo para idosos, verificou que os efeitos da intervenção cognitiva em idosos são promissores, sendo que são de grande importância a prevenção e a reabilitação de consequiências prejudiciais do envelhecimento sobre a qualidade de vida.

A linguagem, sendo um fator que sofre influência de possíveis processos cognitivos em declínio na velhice, é um aspecto que não deve ser negligenciado na intervenção cognitiva do idoso. Tanto este como seus familiares devem conhecer e saber lidar com os processos cognitivos que podem modificar-se com o aumento da idade. Estratégias que visem a intervenção sob aspectos como a memória de trabalho, a atenção e a velocidade de processamento devem ser desenvolvidas para trabalharem a favor da linguagem, utilizando recursos que promovam a valorização do self e que tirem proveito dos pontos fortes da cognição do idoso, como a preservação da memória episódica, o uso de estratégias de compreensão que valorizam o contexto e os aspectos globais do discurso do interlocutor e a habilidade de produzir narrativas interessantes e marcadas pela riqueza na expressão das emoções.

Referências

1 ARBUCKLE, T., GOLD, D. Aging, Inhibition, and Verbosity. Journal of Gerontology: Psychological Sciences, Waltham: v. 48, n. 5, p.225-232, 1993. 2 ARBUCKLE, T., NOHARA-LE CLAIR, M., PUSHKAR, D. Effect of OffTarget Verbosity on Communication Efficiency in a Referential Communication Task. Psychology and Aging, Claremont,: v. 15, n.1, p.65-77, 2000.

3 BALINSKY, B. An Analysis of the Mental Factors of Various Age Groups from Nine to Sixty. Genetic Psychology Monographs, v.23, p.191-234, 1941. 4 BELINCHÓN, M; RIVIÈRE, A., IGOA, J. Psicologia del Lenguaje. Investigacion y Teoría. Madrid: Trotta, 1996.

5 BENEVISTE, E. Problemas de Lingüística Geral I. $4^{\mathrm{a}}$ ed., Tradução de 
Maria da Glória Novak e Maria Luisa Neri. Revisão de Isaac Nicolau Salum. Campinas: Editora da Universidade Estadual de Campinas, 1995.

6 BIELBY, D.; PAPALIA, D. Moral Development and Perceptual Role Taking: their development and interrelationship across the lifespan. International Journal of Aging and Human Development, Denton: v.6, p.293-308, 1975.

7 BIRREN, J.; DEUTCHMAN, D. Guiding Autobiography Groups for Older Adults: exploring the fabric of life. London: The Johns Hopkins University Press, 1991.

8 BODEN, D.; BIELBY, D. The Past as Resource: a conversational analysis of elderly talk. Human Development, Berkeley: v.26, p.308-319, 1983.

9 BURKE, D. Language, Aging, and Inhibitory Deficits: evaluation of a theory. Journal of Gerontology: Psychological Sciences, v. 52B, n. 6, p.254-264, 1997. 10 BURKE, D.; HARROLD, R. Automatic and Effortful Semantic Processes in Old Age: experimental and naturalistic aproaches. In: LIGHT, L.L.; BURKE, D.M. (Eds.). Language, Memory, and Aging. p.100-116. Cambridge University Press, 1988.

11 CARTENSEN, L.; ISAACOWITZ, D.; CHARLES, S. Taking Time Seriously: a theory of socioemotional selectivity. American Psychologist, Washington DC: v. 54, n. 3, p.165-181, 1999.

12 CERELLA, J. Aging and Information Processing Rate. In:. BIRREN, J. E;. SCHAIE, K. W. (Eds.). Handbook of the Psychology of Aging p. 201-221. San Diego: Academic Press, 1990.

13 COHEN, G. Language Comprhension in Old Age. Cognitive Psychology, San Diego: v. 11, p.412-429, 1979.

14 COMMONS, M.; RICHARDS, F.; ARMON, C. Beyond Formal Operations: late adolescent and adult cognitive development. New York: Praeger, 1984.

15 CUNNINGHAM, W.; TOMER, A. Intelectual Abilities and Age: concepts, theories and analyses. In: LOVELACE, E. A. (Ed.). Aging and Cognition: mental processes, self-awareness and interventions. p. 379-406. New York: Elsevier Science Publishers, 1990.

16 GOLD, D.; ANDRES, D.; ARBUCKLE, T.; SCHWARTZMAN.

Measurement and Correlates of Verbosity in Elderly People. Journal of 
Gerontology: Psychological Sciences, v.43, n.2, p.27-33, 1988.

17 GOLD, D.; ARBUCKLE, T. A Longitudinal Study of Off-Target Verbosity. Journal of Gerontology: Psychological Sciences, v.50 B, n.6, p.307-315, 1995. 18 HARRÉ, R., GILLETT, G. The Discursive Mind. Thousand Oaks: Sage Publications, 1994.

19 HORN, J. The Theory of Fluid and Crystallized Intelligence in Relation to Concepts of Cognitive Psychology and Aging in Adulthood. In: CRAIK, F. I.M.; ATREHUB, S. (Eds.). Aging and Cognitive Processes. p.237-278, New York: Plenum, 1982.

20 JAMES, L. Production and Perception of "Verbosity" in Younger and Older Adults. Psychology and Aging, London: v.13, n.3, p.355-367, 1998.

21 JUNCOS-RABADAN, O. Narrative Speech in the Elderly: effects of age and education on telling stories. International Journal of Behavioral Development, v. 19, n. 3, p. 669-685, 1996.

22 KEMPER, S. Language and Aging. In: CRAIK, F.; SALTHOUSE, T. (Eds.). The Handbook of Aging and Cognition. Hillsdale Erlbaum, 1988.

23 KEMTES, K., KEMPER, S. Aging and Resolution of Quantifier Scope Effects. Journal of Gerontology: Psychological Sciences, v. 54B, n. 6, p.350360, 1999.

24 LIGHT, L. Memory and Aging: four hipothesis in search of data. Annual Review of Psychology, Palo Alto: 42, p.333-376, 1991.

25 LOOFT, W. Egocentrism and Social Interaction across the life-span. Psychological Bulletin, v.78, p.73-92, 1972.

26 MAC KAY, D.; ABRAMS; PEDROZA. Aging on the Input Versus Output Side: theoretical implications of age-linked assymetries between detecting versus retrieving orthographic information. Psychology and Aging, Tempe: v. 14, n. 1, p.3-17, 1999.

27 OBLER, L. Language beyond childhood. In: GLEASON, J. B. (Ed.). The Development of Language. p. 275-302, Columbus: Merril Publishing, 1989. 28 ORLANDI, E. O que é Lingüística. São Paulo: Ed. Brasiliense, 1990. 29 PARENTE, M.A.; CAPUANO, A.; NESPOULOUS, J. Ativação de Modelos Mentais no Recontar de Histórias por Idosos. Psicologia: Reflexão e Crítica, Porto Alegre, v. 12, n. 1, p.157-172, 1999. 
30 PARENTE, M.A.; SABOSKINSKI, A. P.; FERREIRA, E.; NESPOULOUS,

J. Memória e Compreensão da Linguagem no Envelhecimento. Estudos Interdisciplinares sobre o Envelhecimento, Porto Alegre, v.1, p.57-76, 1999. 31 PAUL, S. Search for Semantic Inhibition Failure During Sentence Comprehension by Yonger and Older Adults. Psychology and Aging, Claremont: v. 11, n. 1, p.10-20, 1996.

32 PRATT, M., NORRIS, J. The Social Psychology of Aging. Understanding Aging. Cambridge: Blackwell Publishers, 1994.

33 PRETTI, D. A Linguagem dos Idosos: um estudo da análise da conversação. São Paulo: Contexto, 1991.

34 RUBIN, K. The Relationship Between Spatial and Communicative Egocentrism in Children and Young and Old Adults. Journal of Genetic Psychology, Washington DC: 125, p.295-301, 1974.

35 RYAN, E.; LAURIE, S. Evaluations of Older and Younger Adult Speakers: influence of communication effectiveness and noise. Psychology and Aging, Claremont: v.5, p.514-519, 1990.

36 SALTHOUSE, T. Age and Experience Effects on the Interpretation of Orthographic Drawings of Three-Dimensional Objects. Psychology of Aging, Claremont: v.6, p.426-433, 1991.

37 SALTHOUSE, T. The Role of Representativeness in Age Differences in Analogical Reasoning. Psychology and Aging, Claremont: v.2, p.357-362, 1987.

38 SALTHOUSE, T. Speed of Behavior and the Implications for Cognition. In: BIRREN, E.; SCHAIE, K. W. (Eds.). Psychology of Aging. Claremont: p. 400-426, 1985.

39 SEARLE, J. R. Speech Acts. Cambridge: Cambridge University Press, 1969. 40 STINE, E.; WINGFIELD, A. The Assesment of Qualitative Age Differences in Discourse Processing, In: HESS, T. Aging and Cognition: knowledge organization and utilization. North Holland: Elsevier Science Publishers, 1990. 41 STINE, E.; WINGFIELD, A.; POON, L. Speech Comprehension and Memory Through Adulthood: the roles of time and stategy. In: POON, L.W.; RUBIN, D. C.; WILSON, B. (Eds.). Everyday Cognition in Adulthood and Late Life. p. 195-229. New York: Cambridge university Press, 1989. 
42 WOOD, G. Efeitos do Nível de Auto-Eficácia Cognitiva Percebida e de Programas de Treinamento Cognitivo sobre a Capacidade de Memória de Trabalho de Indivíduos Idosos. Belo Horizonte, Dissertação (Mestrado) Programa de Pós-Graduação em Psicologia Social, Universidade Federal de Minas Gerais, Belo Horizonte, 2000.

43 WOODRUFF-PAK, D. The Neuropsychology of Aging. Understanding Aging. Malden: Blackwell Publishers, 1997. 